\title{
DIRECTRICES QUE INFLUYEN EN EL «PROGRAMA DE SEGREGACIÓN EN LA FUENTE $Y$ RECOLECCIÓN SELECTIVA DE RESIDUOS SÓLIDOS» DE LAS MUNICIPALIDADES DE SAN MARTÍN DE PORRES Y ATE, PERÚ (2011-2017)
}

\author{
Keth Lee Jacob Gallegos Blas \\ Patricia Alejandra Nieto Medina \\ Elias Eric Torpoco Beltrán \\ Estudiantes de último año de la carrera de Ingeniería Ambiental en la \\ Universidad Nacional Mayor de San Marcos
}

\begin{abstract}
Resumen: El objetivo del presente estudio fue analizar las directrices que influyen en el Programa de Segregación en la Fuente y Recolección Selectiva de los distritos de San Martín de Porres y Ate, así como la comparación de su evolución de 2011 a 2017 bajo un enfoque descriptivo. Se presenta la descripción de la situación actual del aprovechamiento de residuos sólidos en el país, su manifestación en dispositivos legales y la descripción de los programas de segregación de ambos distritos. Asimismo, un análisis de la cantidad de residuos sólidos recolectados, convenios de gestión y valorización de beneficios ambientales en $\mathrm{kg}$ de $\mathrm{CO}_{2}$ evitados de ambos programas. Como conclusión, se evidencia que Ate mostró una mayor cantidad de residuos sólidos recolectados que San Martín de Porres para el año 2017 en 254,4 toneladas. Asimismo, Ate presentó tres convenios más que San Martín de Porres, lo cual representa una ventaja para adquirir mayores beneficios para el programa. En cuanto a la valorización de beneficios ambientales, Ate superó a San Martín de Porres por $400.543 \mathrm{~kg}$ de $\mathrm{CO}_{2}$. Los resultados meritorios de las tres directrices lograron que Ate se presentara como una municipalidad ejemplar con un programa que tiene una base estratégica con vistas a la sostenibilidad.
\end{abstract}

Palabras claves: residuos sólidos, segregación, directrices, Ate, San Martín de Porres. 


\title{
Influence of Guidelines in the «Programa de Segregación en la Fuente y Recolección Selectiva de Residuos Sólidos» in the Municipality of San Martin De Porres and Ate, Peru (2011-2017)
}

\begin{abstract}
The objective of the research was to analyze the influence of the guidelines in the "Programa de Segregación en la Fuente $y$ Recolección Selectiva» of the districts of San Martin de Porres and Ate; as well as, the comparison of the evolution of each segregation program from 2011 to 2017, using a descriptive approach. The research presented the description of the current situation of the use of solid waste in Peru, the manifestation of recycling at lawful devices, and the description of each segregation program of both districts. Likewise, the research analyzed the amount of solid waste collected, management conventions, and the valuation of environmental benefits in $\mathrm{kg}$ of $\mathrm{CO}_{2}$ avoided from both segregation programs. In conclusion, in 2017 Ate presented a bigger amount of solid waste collected than San Martín de Porres, it was a different of 254,4 tons. Also, Ate presented three more management conventions than San Martín de Porres, which represent an advantage in order to acquire greater benefits for its program. In terms of valuation of environmental benefits, Ate exceeded by $400.543 \mathrm{~kg}$ of $\mathrm{CO}_{2}$ to San Martín de Porres. The great results of the three guidelines analyzed led Ate to present itself as an exemplary municipality with a program that has a strategic base, with a view to sustainability.
\end{abstract}

Keywords: Solid Waste, Segregation, Guidelines, Ate, San Martín de Porres. 
DiRECTRICES QUE INFLUYEN EN EL «PROGRAMA DE SEGREgación EN LA FUENTE Y RECOLECCióN Selectiva de Residuos Sólidos» de las Municipalidades de San Martín de Porres y Ate

\section{Keth Lee Jacob Gallegos Blas}

Estudiante de pregrado del noveno ciclo de la carrera de Ingeniería Ambiental en la Universidad Nacional Mayor de San Marcos. Subcoordinador del Grupo de Estudio de Minería e Impacto Ambiental de la Escuela de Ingeniería Ambiental. Con Diploma Internacional en Medio Ambiente, Seguridad y Salud Ocupacional certificado por la Universidad Nacional de Ingeniería.

Correo electrónico: keth.gallegos@unmsm.edu.pe

\section{Patricia Alejandra Nieto Medina}

Estudiante de pregrado del noveno ciclo de la carrera de Ingeniería Ambiental en la Universidad Nacional Mayor de San Marcos. Coordinadora del Grupo de Estudio de Residuos Sólidos de la Escuela de Ingeniería Ambiental. Pasantía por primeros puestos en la Universidad de Harvard en 2019.

Correo electrónico: patricia.nieto@unmsm.edu.pe

\section{Elias Eric Torpoco Beltrán}

Estudiante de pregrado del noveno ciclo de la carrera de Ingeniería Ambiental en la Universidad Nacional Mayor de San Marcos. Coordinador del Grupo de Estudio de Residuos Sólidos de la Escuela de Ingeniería Ambiental. Con Diploma Internacional en Medio Ambiente, Seguridad y Salud Ocupacional certificado por la Universidad Nacional de Ingeniería.

Correo electrónico: elias.torpoco@unmsm.edu.pe 


\section{Introducción}

En la actualidad, la alta generación e inadecuada disposición de residuos sólidos es uno de los mayores problemas que se presentan a nivel mundial debido al crecimiento demográfico e industrial que provoca la disposición diaria de miles de toneladas de residuos al ambiente, sin tratamiento o manejo previo, originando consecuencias irreversibles (Avendaño 2015). El Banco Mundial (2018) señala que «en el mundo se generan anualmente 2010 millones de toneladas de desechos sólidos municipales, y al menos el $33 \%$ de ellos no se gestionan sin riesgo para el medio ambiente».

Ante este problema, se presenta a la valorización como una solución, que consiste en la búsqueda del aprovechamiento de los residuos sólidos adquiriendo un nuevo valor. Así pues, la adopción de este concepto y «el diseño de un plan de implementación ajustado a las características de la ciudad, le permitirá a la comunidad disponer de un sistema económico y ambientalmente adecuado de manejo de sus residuos» (Ibáñez \& Corroppoli 2002: 45). Para una comunidad, alcanzar estas medidas representa una necesidad que tiene como objetivo lograr modelos de desarrollo ecológicos, conservadores y económicamente independientes, que promueven la creación de fuentes de trabajo, producción de alimentos y la preservación del ambiente (Fernández 2005).

Este concepto no ha sido ajeno a países de América Latina, toda vez que la mayoría de estos tienen como prioridad la valorización de residuos sólidos municipales. En el caso de Perú, el impacto por la inadecuada gestión de los residuos es uno de los causantes del deterioro del ambiente y de los recursos naturales, así como del deterioro de los ambientes urbanos en donde vive cerca del 70\% de la población (Grupo de Trabajo Multisectorial 2008). Ante dicha situación, la valorización de residuos sólidos municipales se ha venido implementando de manera progresiva a través del reciclaje.

El reciclaje ha visto su implementación a través de políticas públicas, tal como el Programa de Segregación en la Fuente y Recolección Selectiva de Residuos Sólidos para gobiernos locales. 
DiRECTRICES QUE INFLUYEN EN EL «PROGRAMA DE SEGREgación EN LA FUENTE Y RECOLECCióN Selectiva de Residuos Sólidos» de las Municipalidades de San Martín de Porres y Ate

se disponen en rellenos sanitarios a través de una cadena formal de reciclaje y un aumento de la conciencia ambiental de los ciudadanos (Rentería \& Zeballos 2014), así como que se «generen empleos verdes, formalizando a los recicladores» (Andina 2017). La Ley de Gestión Integral de Residuos Sólidos, dado a través del decreto legislativo 1278 (2016) y respectivo reglamento mediante el decreto supremo 014-2017-MINAM (2017), establece que las municipalidades distritales deben promover e implementar los programas de segregación en la fuente y recolección selectiva.

En Lima Metropolitana, son varios distritos los cuales han implementado el Programa de Recolección y Segregación en la Fuente de Residuos Sólidos. De todos ellos, Ate es el que ha tenido uno de los mayores protagonismos en cuanto a su cadena de valor de reciclaje, la cual le ha sido merecedora de numerosos reconocimientos, tales como: Buenas Prácticas en Gestión Pública 2014 (CAD)-Gestión ambiental efectiva, Premio Nacional Ambiental 2014 (MINAM)-Ecoeficiencia en instituciones estatales, Gestión Ambiental Sostenible 2014 (MINAM), Municipio con mayor recolección de vidrio 2014 (FUNDADES), Buenas Prácticas en Gestión Pública 2015 (CAD)-Cooperación público privada y Premio a la Innovación Municipal 2016-Desarrollo Económico Social (Municipalidad de Ate 2017), así como le ha permitido otorgar oportunidades de trabajo a población vulnerable (Espejo 2016). Asimismo, desde que empezó el Programa de Incentivos en Gestión Municipal, dado por el Ministerio de Economía y Finanzas, Ate ha cumplido todos los años con la meta establecida de implementación del programa de segregación a un determinado porcentaje de población.

De otro lado se encuentra el distrito de San Martín de Porres, cuyo programa ha tenido una evolución distinta, no llegando a cumplir una meta establecida de implementación del programa de segregación en un año, así como inconvenientes en cuanto a su gestión. Todo ello a pesar de asemejarse al distrito de Ate en cuanto a habitantes, ya que su población equivale al 83\% de la población de San Martín de Porres; su generación per cápita (GPC), que se diferencia en 1,8\% menos en relación con el GPC de Ate; y la clasificación municipal tipo C, que les otorga la misma meta en el Programa de Incentivo de Gestión Municipal. Estas diferencias se deben al desarrollo de las directrices en la gestión municipal, que han conllevado a resultados diferentes. Debido a ello nace el interés de realizar el presente estudio, a través de la revisión de información bibliográfica, con el objetivo de analizar las directrices que influyen en el Programa de Segregación de la Fuente y Recolección Selectiva de ambos distritos y comparar su evolución de 2011 a 2017. 


\section{Revisión bibliográfica}

A través de una metodología descriptiva y revisión bibliográfica se encuentra que la situación que atraviesa el país respecto a la valorización de residuos sólidos ha ido evolucionando a través del tiempo. Se presume que el primer tipo de valorización en el Perú es el reciclaje. Según Mulato (2019), la línea del reciclaje en el país comenzó a raíz del fenómeno migratorio a inicios de los años setenta, que trajo consigo una mayor demanda de bienes y servicios; asimismo, el incremento de la tasa de natalidad en los noventa conllevó a un aumento de asentamientos humanos y construcciones, entre otros, y, en consecuencia, a una generación mayor de residuos sólidos. Ante la insuficiente capacidad operativa de las municipalidades, surgen los llamados recicladores informales, para luego ir aumentando la actividad del reciclaje a través de ellos. Tiempo después, un reducido número de municipalidades a nivel nacional implementaron un manejo selectivo de sus residuos, tal es el caso de Villa El Salvador, el cual implementa en 2005 el Programa Municipal de formalización de recicladores Bono Verde, otorgando un descuento económico en el pago de arbitrios a las casas que segregan y ayudan a los recicladores. Para 2009, a nivel nacional los recicladores llegaron a recolectar 292.636,94 toneladas de residuos (Ruiz 2010).

Posterior a ello, la valorización ha ido creciendo hasta plasmarse por medio de dispositivos legales. Uno de ellos se da a través del Programa de Incentivos en la Gestión Municipal, el cual se creó mediante la ley 29332 en el año 2009, entrando en vigor el año 2010. Se define como una herramienta vinculada al presupuesto por resultado que está a cargo del Ministerio de Economía y Finanzas, cuyo objetivo es promover la mejora continua de los servicios públicos que brindan las municipalidades (Ministerio de Economía y Finanzas s.f.).

Dentro del Programa de Incentivos se especifican metas, las cuales deben ser cumplidas por un periodo determinado. Una de ellas es el Programa de Segregación en la Fuente y la Recolección Selectiva, la cual fue impulsada desde 2011, llegando a ser una de las herramientas más importantes para la valorización de residuos sólidos a nivel nacional. Cada año, las metas vinculadas al programa incrementan gradualmente, en su comienzo se implementó el programa en un 5\% de la población, en 2012 en un 7\%, en 2013 un 10\%, en 2014 un 20\%, en 2015 el porcentaje se rigió según la clasificación del distrito, y para 2017 y 2018 se rigió según el reporte de la cantidad de residuos sólidos inorgánicos municipales recuperados (Mulato 2019). De acuerdo con Huiman (2017), el «extender los incentivos municipales al ciudadano y a las empresas motivando su participación en los Programas de Recolección Selectiva y Segregación en la Fuente» es uno de los pasos para un camino propicio hacia el reciclaje. 
DiRECTRICES QUE INFLUYEN EN EL «PROGRAMA DE SEGREgación EN LA FUENTE Y RECOLECCióN Selectiva de Residuos Sólidos» de las Municipalidades de San Martín de Porres y Ate

No obstante, hoy en día se observa que la tasa de valorización es poca. Según el Ministerio del Ambiente (MINAM 2018), en el año 2016 se generaron 7.005.576 toneladas de residuos domiciliarios, de los cuales solo el 1,9\% de los residuos aprovechables se reciclaron, muchos de estos gracias al programa de segregación en la fuente, significando que otro porcentaje se fue a rellenos sanitarios, botaderos, ríos u océanos, provocando un impacto negativo al ambiente y una pérdida de potencial de valorización. La informalidad también es un aspecto que está presente en todo el Perú, ejemplificando así que los recicladores, actores esenciales de la pirámide del reciclaje, tienen un modo de trabajo informal en el país equivalente al 86,62\%, predominando mayoritariamente frente al formal (Municipalidad Metropolitana de Lima 2014). Pese a la formalización de los recicladores, en muchos casos, las cantidades de residuos sólidos recolectados durante el programa se ven desviados por este aspecto, no registrando el verdadero potencial.

El principal generador de residuos sólidos a nivel nacional es Lima Metropolitana, debido a que genera el 45\% del total producido a nivel nacional y solo el 4\% se recicla (Sandoval 2016), a pesar de que varios distritos implementaron y cumplieron el programa de segregación según las metas establecidas a través de los años (tabla 1). Asimismo, según los estudios realizados por Lima Cómo Vamos (2019), dentro de los principales problemas ambientales en Lima, según sus pobladores, está la gestión de residuos sólidos, la cual engloba el sistema de recojo de residuos $(35,8 \%)$ y la falta de sistema de reciclaje $(31,3 \%)$.

En efecto, el primero trae como consecuencia la existencia de 631 puntos críticos de residuos sólidos en Lima Metropolitana y Callao, siendo algunos de los principales distritos: San Juan de Miraflores, Villa el Salvador, Villa María del Triunfo, Comas y San Martín de Porres (Defensoría del Pueblo 2019), y de lo último se puede inferir que existe un posible incumplimiento por parte de las municipalidades o existen directrices que provocan un deterioro de su rendimiento. Latorre (2016: 10) afirma que «si bien es positivo el porcentaje de municipalidades que tienen el servicio de recojo, aún no se ha podido lograr reaprovechar correctamente los residuos sólidos generados». 
Tabla 1.

Cumplimiento del programa de segregación de las municipalidades de Lima Metropolitana 2011-2017

\begin{tabular}{|c|c|c|c|c|c|c|c|}
\hline DISTRITO & 2011 & 2012 & 2013 & 2014 & 2015 & 2016 & 2017 \\
\hline Ancón & No & Sí & Sí & Sí & Sí & Sí & No \\
\hline Ate & Sí & Sí & Sí & Sí & Sí & Sí & Sí \\
\hline Barranco & No & Sí & Sí & Sí & Sí & No & No \\
\hline Breña & No & Sí & Sí & Sí & Sí & Sí & Sí \\
\hline Carabayllo & Sí & Sí & Sí & Sí & Sí & Sí & Sí \\
\hline Chaclacayo & Sí & Sí & Sí & Sí & No & Sí & Sí \\
\hline Chorrillos & No & Sí & No & No & No & No & No \\
\hline Cieneguilla & Sí & Sí & Sí & Sí & Sí & Sí & No \\
\hline Comas & Sí & Sí & Sí & Sí & Sí & Sí & Sí \\
\hline El Agustino & Sí & Sí & Sí & Sí & Sí & Sí & No \\
\hline Independencia & Sí & Sí & Sí & Sí & Sí & Sí & Sí \\
\hline Jesús María & Sí & Sí & Sí & Sí & Sí & Sí & Sí \\
\hline La Molina & Sí & Sí & No & No & Sí & Sí & Sí \\
\hline La Victoria & Sí & Sí & Sí & Sí & Sí & Sí & Sí \\
\hline Lince & Sí & Sí & Sí & No & Sí & Sí & No \\
\hline Los Olivos & Sí & Sí & Sí & Sí & Sí & Sí & Sí \\
\hline Lurigancho & Sí & Sí & No & Sí & Sí & Sí & Sí \\
\hline Lurín & Sí & Sí & Sí & Sí & No & Sí & Sí \\
\hline Magdalena del Mar & Sí & Sí & No & Sí & Sí & Sí & No \\
\hline Miraflores & Sí & Sí & Sí & Sí & Sí & No & Sí \\
\hline Pachacamac & Sí & Sí & No & Sí & Sí & No & Sí \\
\hline Pucusana & No & Sí & Sí & Sí & Sí & No & No \\
\hline Pueblo Libre & - & Sí & Sí & Sí & NO & Sí & Sí \\
\hline Puente Piedra & No & Sí & Sí & Sí & Sí & Sí & No \\
\hline Punta Hermosa & No & Sí & Sí & No & Sí & No & No \\
\hline Punta Negra & Sí & Sí & No & No & Sí & No & No \\
\hline Rímac & Sí & Sí & Sí & No & Sí & Sí & No \\
\hline San Bartolo & No & No & Sí & Sí & Sí & Sí & Sí \\
\hline San Borja & Sí & Sí & Sí & No & No & No & Sí \\
\hline San Isidro & No & No & No & No & Sí & Sí & Sí \\
\hline $\begin{array}{l}\text { San Juan de } \\
\text { Lurigancho }\end{array}$ & Sí & Sí & Sí & Sí & Sí & No & Sí \\
\hline $\begin{array}{l}\text { San Juan de } \\
\text { Miraflores }\end{array}$ & Sí & Sí & Sí & Sí & Sí & Sí & Sí \\
\hline San Luis & Sí & Sí & Sí & Sí & No & No & No \\
\hline San Martín de Porres & Sí & Sí & Sí & Sí & Sí & Sí & No \\
\hline San Miguel & Sí & Sí & No & Sí & Sí & Sí & Sí \\
\hline
\end{tabular}


DiRECTRICES QUE INFLUYEN EN EL «PROGRAMA DE SEGREgación EN LA FUENTE Y RECOLECCióN Selectiva de Residuos Sólidos» de las Municipalidades de San Martín de Porres y Ate

\begin{tabular}{|l|c|c|c|c|c|c|c}
\multicolumn{1}{|c|}{ DISTRITO } & $\mathbf{2 0 1 1}$ & $\mathbf{2 0 1 2}$ & $\mathbf{2 0 1 3}$ & $\mathbf{2 0 1 4}$ & $\mathbf{2 0 1 5}$ & $\mathbf{2 0 1 6}$ & $\mathbf{2 0 1 7}$ \\
\hline Santa Anita & Sí & Sí & Sí & Sí & Sí & Sí & Sí \\
\hline Santa María del Mar & Sí & Sí & Sí & No & No & No & No \\
\hline Santa Rosa & Sí & Sí & Sí & Sí & No & No & No \\
\hline Santiago de Surco & Sí & Sí & Sí & Sí & No & Sí & Sí \\
\hline Surquillo & Sí & Sí & No & No & No & No & Sí \\
\hline Villa El Salvador & Sí & Sí & No & Sí & Sí & No & Sí \\
\hline $\begin{array}{l}\text { Villa María del } \\
\text { Triunfo }\end{array}$ & Sí & Sí & Sí & Sí & No & Sí & Sí \\
\hline
\end{tabular}

Elaboración propia en base a Ministerio de Economía y Finanzas (s.f.).

De las principales municipalidades distritales en Lima Metropolitana que han implementado el Programa de Segregación en la Fuente y Recolección Selectiva de Residuos Sólidos, se tiene a Ate y San Martín de Porres, ambas poseen similitudes en cuanto a número de población, GPC y clasificación municipal tipo C (este último establece la misma meta para las dos municipalidades en el Programa de Incentivos en la Gestión Municipal desde sus inicios). Sin embargo, manifiestan realidades diferentes con relación a la evolución, resultados meritorios y cumplimiento de metas del programa de segregación.

En primer lugar, está el distrito de San Martín de Porres, ubicado en Lima Norte. Según el Instituto Nacional de Estadística (INEI) (2007), en su Censo Nacional realizado en el año 2007, se registró un total de 579.561 habitantes, siendo su GPC de 0,611 kg/hab/día (según su Estudio de Caracterización de Residuos Sólidos 2016). Su generación de residuos sólidos domiciliarios ha aumentado a través de los años (gráfico 1), siendo la disposición de sus residuos en los rellenos sanitarios de Huaycoloro y Zapallal hasta 2014, posterior a ese año, la disposición se realiza, hasta la actualidad, en Zapallal y Modelo de Callao (INEI 2015, 2019). 
Gráfico 1.

Residuos sólidos domiciliarios generados en San Martín de Porres 2011-2017

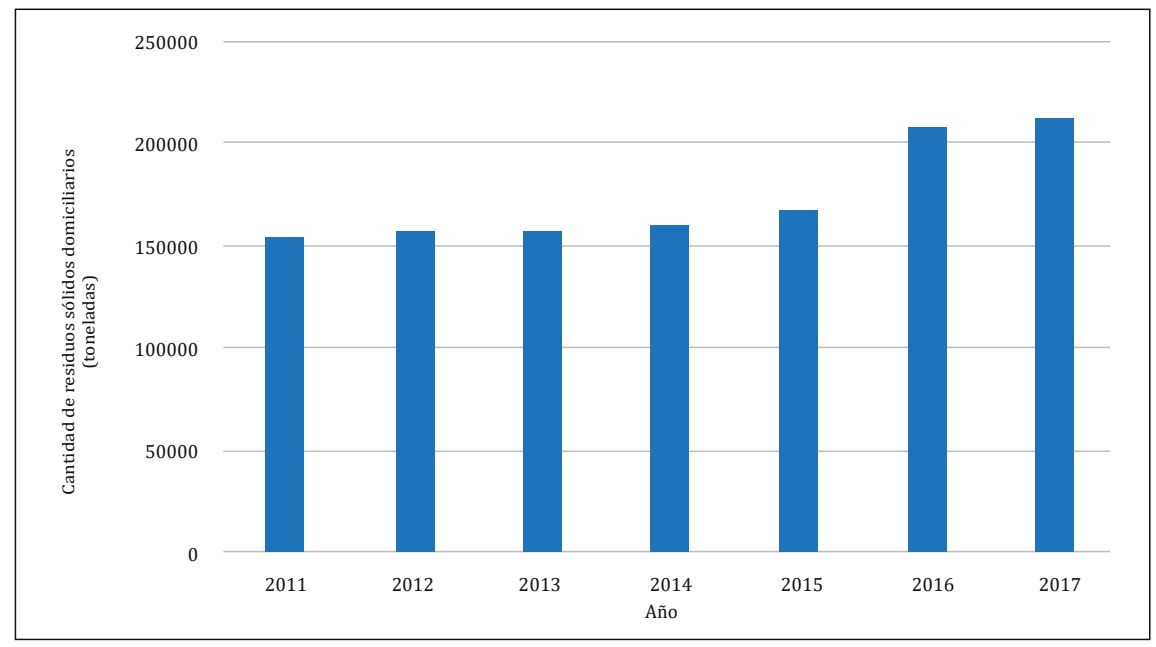

Elaboración propia en base a INEI (2015 y 2019).

La implementación de su programa comenzó en el año 2011, y actualmente es denominado «Recicla y Emprende», cuyos actores involucrados son las juntas vecinales, asociación de recicladores, empresas privadas, instituciones educativas y demás. El programa se dio a conocer por medio de charlas informativas, entrega de trípticos, volantes, stickers, almanaques, realizado por los promotores y sensibilizadores ambientales que a su vez registraron a la población interesada para, en 2012, empezar con la recolección.

La sensibilización es realizada por los promotores ambientales y la recolección de residuos mediante recicladores, con quienes se estableció un vínculo para admitir su formalidad y puedan realizar dichas actividades. Al 2015secuentacon seisasociaciones: Asociación de recicladoresguardianes del medio ambiente, Asociación de recicladores protectores del medio ambiente y la salud pública, Asociación de recicladores en defensa de la ecología y del medio ambiente, Asociación de recicladores veladores del hábitat y la vida, Asociación de recicladores Sol Naciente y Asociación de recicladores Nueva Vida. El transporte se realiza mediante triciclos a través de los once sectores que cubren todo el distrito, distribuyéndose proporcionalmente cada sector por asociación de recicladores. Además, anteriormente, ellos se hacían cargo de la segregación de los residuos recolectados y de la comercialización mediante coordinaciones directas con la empresa papelera El Bosque S.R.L., 
DiRECTRICES QUE INFLUYEN EN EL «PROGRAMA DE SEGREgación EN LA FUENTE Y RECOLECCióN Selectiva de Residuos Sólidos» de las Municipalidades de San Martín de Porres y Ate

la cual tenía establecido un convenio con la municipalidad con anterioridad. Sin embargo, estas acciones de comercialización conllevaron problemas con los datos registrados de residuos recolectados, de modo que en 2014 se implementó una planta de segregación, donde se empezó a tener un mejor control logístico por parte de la municipalidad.

En segundo lugar, se tiene al distrito de Ate, el cual está ubicado en Lima Este, contando con una población de 478.278 habitantes según el Censo Nacional de 2007 (INEI 2007) y con una GPC de 0,622 kg/hab/día (Municipalidad de Ate 2017). La generación de residuos sólidos en este distrito ha variado en el tiempo (gráfico 2), con aumentos y disminuciones, asimismo, la disposición de sus residuos sólidos se realiza en el relleno sanitario de Huaycoloro, hasta la fecha (INEI 2015 y 2019).

\section{Gráfico 2:}

\section{Residuos sólidos domiciliarios generados en Ate 2010-2017}

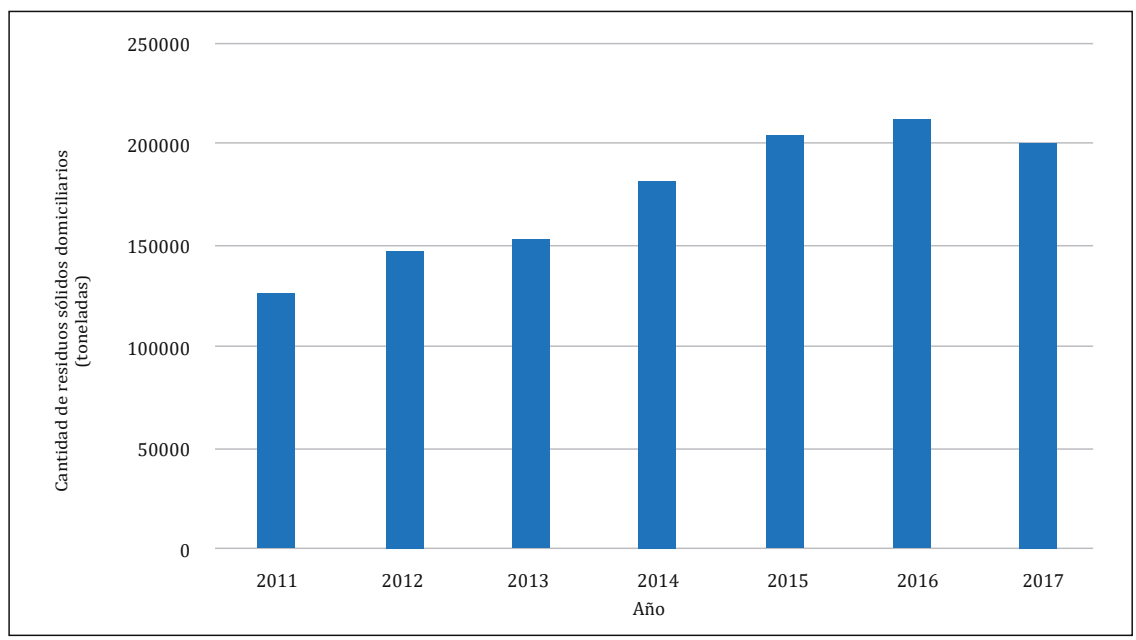

Elaboración propia en base a INEI (2015 y 2019).

Su programa comenzó en el año 2011 con 4 trabajadores, para 2015 esta cifra aumentó a más de 50 colaboradores (Recíclame Perú 2015); asimismo, los actores involucrados son los vecinos, recicladores formalizados, instituciones educativas públicas y privadas, así como empresas públicas y privadas, que trabajan de manera articulada para lograr una adecuada gestión de residuos sólidos municipales.

La sensibilización se realiza por parte de los promotores ambientales, mientras que la recolección es realizada por los recicladores formalizados 
con una frecuencia semanal en cinco zonas. Para el transporte de los residuos se usan coches acondicionados, triciclos u otros vehículos, que trasladan los residuos al centro de acopio o hacen una transferencia a un vehículo de mayor capacidad. Finalmente, una vez reunidos todos los residuos en el centro de acopio, se emplean equipos y maquinarias para realizar las actividades de separación, clasificación, minimización del volumen y almacenamiento, en el trabajo cuentan con todas las medidas de seguridad e higiene. La comercialización es de forma indirecta, porque interviene la municipalidad con recicladores formalizados dependientes y empresas privadas a través de convenios de gestión, siendo estas: Empresa Gexim S.A.C., Empresa OwensIllinois Perú S.A., Empresa Praxis Ecology S.A.C., Empresa Tetrapack.

\section{Discusión}

De acuerdo al censo realizado por el INEI el año 2007, Ate posee aproximadamente el 83\% de la población de San Martín de Porres, pero por el tipo de comportamiento de consumo y generación de residuos, San Martín de Porres tiene una generación per cápita menor en un 1,8\% en relación a la GPC de Ate, por lo cual se compensa relativamente en la cantidad total de residuos sólidos generados a nivel distrital. Por tanto, se puede considerar de manera macro que ambas poblaciones manejan cantidades similares de residuos; además, presentan la misma clasificación municipal, lo que los direcciona a la misma meta en el Programa de Incentivos en la Gestión Municipal.

Por lo expuesto anteriormente, se demuestra la similitud entre ambos distritos en este aspecto, pero la implementación del Programa de Segregación en la Fuente y Recolección Selectiva ha conllevado a resultados diferentes; esto debido a la gestión de sus directrices, las cuales son: la cantidad de residuos sólidos recolectados, el número de convenios de gestión y los kilogramos de dióxido de carbono evitados por tonelada de estos mismos ( $\mathrm{kg}$ de $\mathrm{CO}_{2 / \mathrm{tn}}$ residuo recolectado), descritos a continuación.

\subsection{Cantidad de residuos sólidos recolectados}

En primer lugar, la cantidad de residuos sólidos recolectados, como se observa en el gráfico 3, es mayor por parte de Ate, llegando a la cantidad máxima de 988,85 toneladas de residuos sólidos, en cambio San Martín de Porres llega a 734,45 toneladas, ambos para el año 2017. 
DiRECTRICES QUE INFLUYEN EN EL «PROGRAMA DE SEGREgación EN LA FUENTE Y RECOLECCióN Selectiva de Residuos Sólidos» de las Municipalidades de San Martín de Porres y Ate

\section{Gráfico 3. \\ Cantidad de residuos sólidos recolectados durante el Programa de Segregación en la Fuente y Recolección Selectiva de ambos distritos}

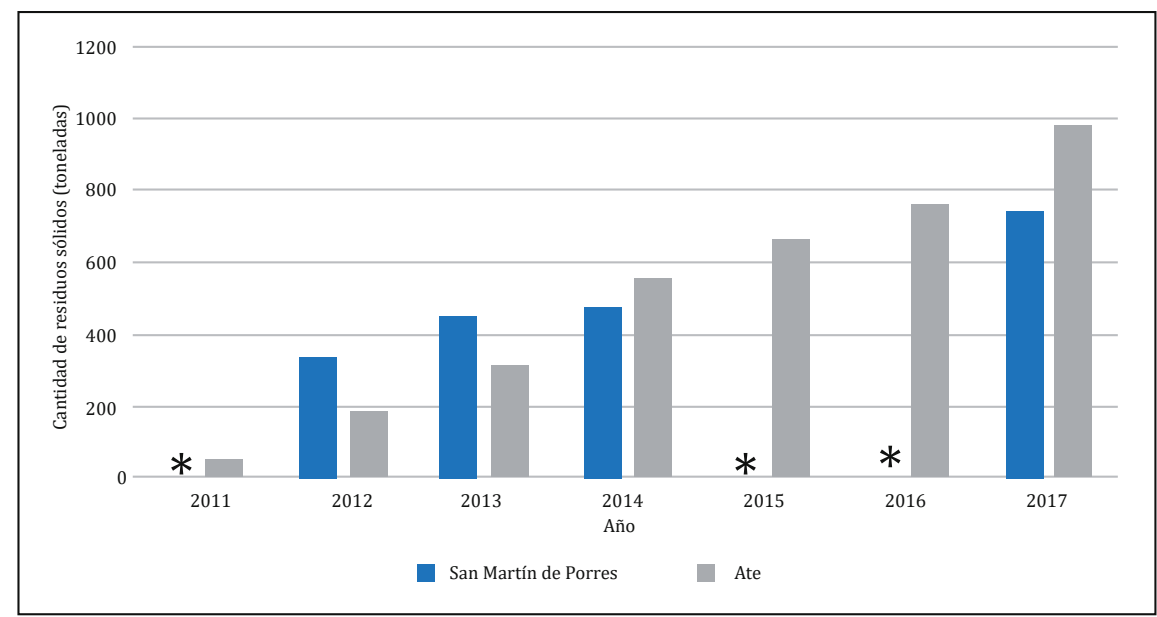

* La municipalidad no muestra registro de la cantidad de residuos sólidos recolectados en los años 2011, 2015 y 2016.

Elaboración propia en base a Municipalidad de Ate (2016 y 2017) y Municipalidad de San Martín de Porres (2016 y 2018).

Esta diferencia se puede deber al aspecto educacional representado en las campañas de sensibilización. Por un lado, la Municipalidad de San Martín de Porres ejecuta las campañas a través de una difusión y sensibilización durante algunos días, según el Calendario Ambiental Peruano, así como visitas a los domicilios por parte de los promotores y sensibilizadores ambientales. Del otro lado, la Municipalidad de Ate manifiesta una mayor campaña de sensibilización, la cual se viene realizando a través de tres estrategias de comunicación permanente: la primera, de manera indirecta, mediante materiales de difusión (volantes, trípticos, entre otros); la segunda, de manera directa, a través de actividades concatenadas por medio de talleres informativos y de participación, para luego realizar la visita a los domicilios, reforzándose mediante la participación en eventos según el Calendario Ambiental Peruano; y, por último, la difusión por medios de comunicación local. Esto último ha conllevado a una mayor cobertura de predios inscritos, empadronándose un total de 47.580 en 2016, a comparación de San Martín que tan solo presenta 39.002 para 2015 .

Otro aspecto que influye en la cantidad de residuos sólidos recolectados es el tipo de transporte usado. En San Martín de Porres se da mediante vehículos no convencionales (triciclo), lo cual limita su capacidad, mientras que en Ate se da por medio de vehículos no convencionales (triciclos) 
y convencionales (camión baranda, camioneta, coches de recolección y motokar), y por ello muestra mayor eficiencia al recolectar los residuos, contando con vehículos acondicionados para una mayor capacidad de carga.

Asimismo, la infraestructura y el consecuente manejo de registros es un factor importante ya que representa un control logístico y veracidad dela cantidad de residuos sólidos recolectados, permitiendo una correcta representación de la evolución del programa en las dos municipalidades a analizar.

Por un lado, Ate cuenta con un centro de acopio y planta de reciclaje, el cual posee instalaciones tales como oficinas, comedor y sala de reuniones, además cuenta con todos los equipos necesarios para realizar el trabajo. En consecuencia, el manejo de registros lo realiza de dos maneras: la indirecta, por medio de los recicladores independientes, y la directa, que está dada por la misma municipalidad, no manifestando inconvenientes en cuanto a ello.

Del otro lado, San Martín de Porres no presentó infraestructura adecuada hasta 2014 y el manejo solo se realiza de manera indirecta a través de los mismos recicladores formalizados, quienes realizaban la comercialización. Esto presentó inconvenientes y la municipalidad manifestó: «no todos los recicladores venden sus residuos reaprovechables en empresas formales por causas relacionadas a la diferencia de precio, distancia [...], algunos prefieren vender sus residuos reaprovechables en otros centros de acopio que no son necesariamente formales» (Municipalidad de San Martín de Porres 2016: 18).

Asimismo, la diferencia de la cantidad de residuos sólidos recolectados por ambos distritos está influenciado por la informalidad. En Lima Norte, donde se ubica San Martín de Porres, según el Plan Integral para la Gestión de Residuos Sólidos para Lima Metropolitana (2014), la cadena de recolección realizada por recicladores formales es del 2,3\% con 7,23 t/día, mientras que el informal fue de 16,7\% con 356,08 t/día (Huiman 2018). De esta manera, cierta cantidad de residuos potencialmente aprovechables son desviados antes de la recolección del programa de segregación debido a la alternativa de recolección informal no previsto por la gestión municipal.

\subsection{Convenios de gestión}

En cuanto a la creación de convenios de gestión entre las municipalidades y empresas comercializadoras, San Martín de Porres posee solo un convenio con la empresa papelera El Bosque S.R.L. Este convenio tiene como únicos beneficiarios a la empresa y las asociaciones de recicladores debido a que la comercialización directa de residuos sólidos recolectados se realiza entre ellos. En cambio, Ate estableció cuatro convenios con empresas (Gexim S.A.C., Owens-Illinois Perú S.A., Praxis Ecology S.A.C. y Empresa Tetrapack), llamadas alianzas público-privadas. Eso es de vital importancia 
DiRECTRICES QUE INFLUYEN EN EL «PROGRAMA DE SEGREgación EN LA FUENTE Y RECOLECCióN Selectiva de Residuos Sólidos» de las Municipalidades de San Martín de Porres y Ate

para el programa, ya que es una estrategia de inversión que provee de múltiples beneficios logísticos mediante una relación recíproca en la que la municipalidad brinda a las empresas los residuos sólidos recolectados por las asociaciones de recicladores para agregarlo como insumo a sus procesos productivos como material reciclable, y la empresa otorga bienes, tales como: equipo de protección personal, indumentaria para los operarios y bolsas; además otorgan servicios, tales como: equipos para el centro de acopio, alquiler del local y materiales de difusión. Todo ello hace factible el desarrollo del programa y ha permitido «revertir directamente los gastos operativos del programa» (Municipalidad de Ate 2016).

\subsection{Kilogramos de dióxido de carbono evitados por tonelada de estos mismos ( $\mathrm{kg} \mathrm{de} \mathrm{CO}_{2} / \mathrm{tn}$ residuo recolectado)}

Existe, además, una comparación en cuanto a la valorización de beneficios ambientales en kilogramo de $\mathrm{CO}_{2}$ evitados durante el Programa de Segregación en la Fuente y la Recolección Selectiva para ambos distritos. Según el gráfico 4, para el año 2017, Ate evitó un total de $938.238 \mathrm{~kg}$ de $\mathrm{CO}_{2}$, mientras que San Martín de Porres, 537.694 kg de $\mathrm{CO}_{2}$.

Gráfico 4.

Emisiones de $\mathrm{CO}_{2}$ evitados por los residuos sólidos reciclados durante el programa en el año 2017

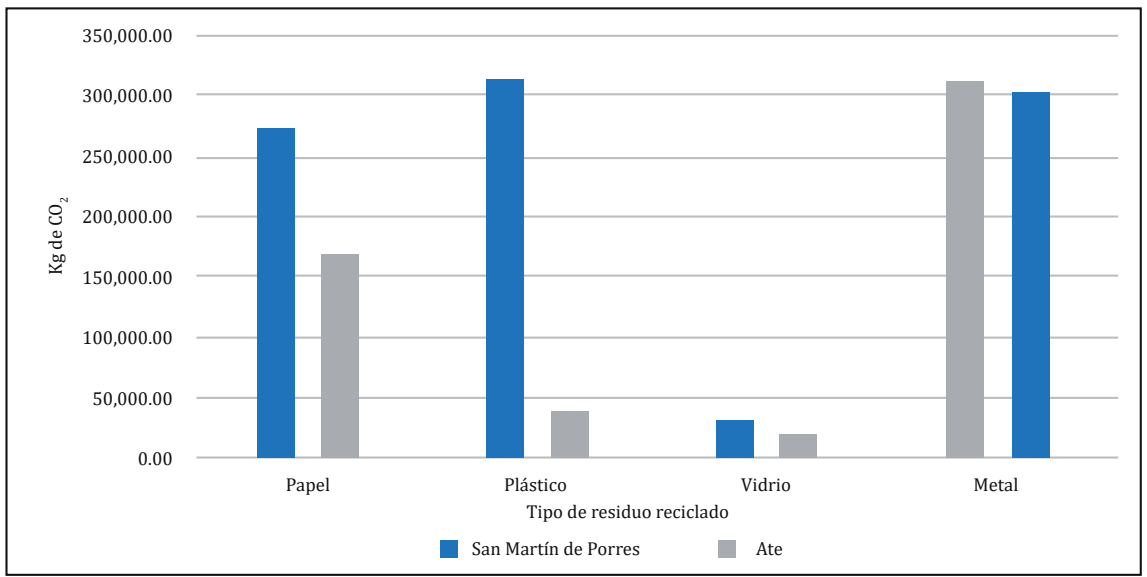

Elaboración propia.

El MINAM (2015) establece los beneficios ambientales reflejados en kg de $\mathrm{CO}_{2}$ evitados según la clasificación de residuos (tabla 2), por ende, la diferencia de $\mathrm{kg}$ de $\mathrm{CO}_{2}$ evitados entre ambos distritos guarda relación con los tipos de residuos sólidos recolectados. 
Tabla 2.

Consumo de emisiones de $\mathrm{CO}_{2}$ por residuo

\begin{tabular}{l|l} 
Residuos reciclados & Beneficios \\
\hline Reciclar 1 tonelada de papel & Se evita emitir $820 \mathrm{~kg} \mathrm{de} \mathrm{CO}_{2}$ \\
\hline Reciclar 1 tonelada de plástico & Se evita emitir $410 \mathrm{~kg} \mathrm{de} \mathrm{CO}_{2}$ \\
\hline Reciclar 1 tonelada de vidrio & Se evita emitir $180 \mathrm{~kg} \mathrm{de} \mathrm{CO}_{2}$ \\
\hline Reciclar 1 tonelada de metal & Se evita emitir $2.000 \mathrm{~kg} \mathrm{de} \mathrm{CO}_{2}$ \\
Elaboración propia en base a MINAM (2015). &
\end{tabular}

Ate, en el año 2017, presentó una recolección de 337,37 toneladas de papel, 774,75 toneladas de plástico, 172,36 toneladas de vidrio y 156,46 toneladas de metal; por otro lado, San Martín de Porres presentó 207,96 toneladas de papel, 99,52 toneladas de plástico, 101,8 toneladas de vidrio y 154,02 toneladas de metal, respectivamente. En consecuencia, se observa una mayor cantidad de $\mathrm{kg}$ de $\mathrm{CO}_{2}$ evitados en papel y plástico por parte de Ate, debido a que, a diferencia de San Martín, recolectó más residuos de ese tipo, sin embargo, en metal y vidrio la cantidad es similar en ambos.

Mediante la información mostrada anteriormente, se comparó la evolución de ambas municipalidades en condiciones similares, que llegaron a resultados diferentes debido a las medidas que tomaron durante su gestión. Esto a través del análisis de directrices, las cuales fueron: la cantidad de residuos sólidos recolectados, influenciado por el aspecto educacional; la capacidad de carga de vehículos de recolección y el manejo de registro de los residuos; los convenios de gestión que resultan en beneficios para el programa; y la valorización de beneficios ambientales en cantidad de kg de $\mathrm{CO}_{2}$ evitados que dependen del tipo de residuo recolectado. Por consiguiente, el adecuado y óptimo desarrollo de estas directrices es la clave para desarrollar un programa sostenible y exitoso, tal como se presenta en el caso de Ate.

\section{Conclusiones}

Los resultados del estudio señalan que el análisis de la directriz de cantidad de residuos sólidos recolectados a través de los años, para el caso del distrito de San Martín de Porres, registró un aumento paulatino, mientras que para el caso de Ate presentó un aumento progresivo, llegando a sobrepasar a San Martín de Porres en el año 2017 en 254,4 toneladas. Respecto a la directriz de número de convenios, Ate presentó cuatro y San Martín solo uno, siendo el primero el que posee mayores beneficios para el programa. Asimismo, en 
DiRECTRICES QUE INFLUYEN EN EL «PROGRAMA DE SEGREgación EN LA FUENTE Y RECOLECCióN Selectiva de Residuos Sólidos» de las Municipalidades de San Martín de Porres y Ate

la valoración de beneficios ambientales, Ate superó a San Martín de Porres por $400.543 \mathrm{~kg} \mathrm{de} \mathrm{CO}_{2}$ debido a una mayor cantidad recolectada de papel y plástico.

Elcrecimiento paulatino dela cantidad deresiduos sólidos recolectados en San Martín de Porres se debió al impacto leve del aspecto educacional en la población, que en consecuencia registró un menor número de predios inscritos respecto de Ate, ciertos inconvenientes en el registro de residuos recolectados, una carencia previa de infraestructura adecuada, así como la influencia de la informalidad. Por otro lado, el crecimiento de residuos sólidos recolectados en Ate fue progresivo, lo cual se debió a un aspecto educacional estructurado por parte de la municipalidad, así como la variabilidad de medios de transporte, tanto convencional como no convencional, que resulta en mayor capacidad de recolección de residuos sólidos, y la presencia de una infraestructura adecuada para el almacenamiento, recolección y segregación de residuos.

La existencia de un solo convenio con una empresa por parte de San Martín de Porres ha conllevado solo al beneficio económico para las asociaciones de recicladores, mientas que en Ate la creación de cuatro convenios bajo la modalidad de alianzas público-privadas da como resultado beneficios logísticos para la municipalidad, lo cual permite un ahorro en los gastos operativos del programa, garantizando así su sostenibilidad y permitiendo proyectar el servicio a toda la población en los años siguientes.

Respecto a la valorización de beneficios ambientales, Ate evitó mayor cantidad de kg de $\mathrm{CO}_{2}$ que San Martín de Porres, traduciéndose en una mayor contribución a la conservación del ambiente. La diferencia de $400.543 \mathrm{~kg}$ de $\mathrm{CO}_{2}$ entre ambos distritos se debió a la cantidad recolectada de un tipo de residuo sólido, ya que cada tipo posee una unidad equivalente en $\mathrm{kg}$ de $\mathrm{CO}_{2}$, por lo que la mayor cantidad de papel y plástico recolectado por Ate marcó la diferencia.

El análisis de la influencia de las directrices en el Programa de Segregación en la Fuente y Recolección Selectiva de Ate, llevó a que el programa sea más exitoso y se presente como un municipio ejemplar, simbolizando el cumplimiento meritorio al recibir diversos premios y reconocimientos por su programa, tales como: Buenas Prácticas en Gestión Pública 2014 (CAD)-Gestión ambiental efectiva, Premio Nacional Ambiental 2014 (MINAM)-Ecoeficiencia en instituciones estatales, Gestión Ambiental Sostenible 2014 (MINAM), Municipio con mayor recolección de vidrio 2014 (FUNDADES), Buenas Prácticas en Gestión Pública 2015 (CAD)-Cooperación público privada y Premio a la Innovación Municipal 2016-Desarrollo Económico Social (Municipalidad de Ate 2017).

Ante lo mencionado, el estudio sugiere tomar como modelo los lineamientos de la gestión del Programa de Segregación en la Fuente y 
Recolección Selectiva de Residuos Sólidos que realiza la Municipalidad de Ate para diseñar, reforzar y/o adecuar el programa en otras municipalidades, y así cumplir con las metas establecidas del Programa de Incentivos de la Gestión Municipal mediante los resultados meritorios en las directrices analizadas, los cuales, a su vez, se encuentran alineados con los Objetivos de Desarrollo Sostenible adoptados por la Asamblea General de las Naciones Unidas (por ejemplo, el objetivo 13 «Acción por el Clima», el objetivo 8 «Trabajo decente y crecimiento económico» y el objetivo 17 «Alianza para lograr los objetivos»). Por otro lado, estos resultados brindan mayor confiabilidad en apostar por el establecimiento de alianzas público-privadas en el marco de la gestión municipal, ya que hay seguridad por parte de la empresa privada de ofrecer servicios y/o bienes mediante mecanismos de canje y hace rentable la actividad del reciclaje.

\section{Referencias}

Andina (3 de junio 2017). Más de 3,000 recicladores se formalizaron gracias al programa de incentivos municipales. Andina, sección Actualidad. Recuperado el 23 de agosto de 2020. https://andina.pe/agencia/ noticia-mas-3000-recicladores-se-formalizaron-gracias-al-programaincentivos-municipales-669468.aspx

Avendaño, E. (2015). Panorama actual de la situación mundial, nacional y distrital de los residuos sólidos: análisis del caso Bogotá D.C. Programa Basura Cero (tesis de pregrado para optar al grado de Ingeniero Ambiental). Universidad Nacional Abierta y a Distancia, Bogotá, Colombia. https:// repository.unad.edu.co/handle/10596/3417

Banco Mundial (20 de septiembre de 2018). Los desechos: un análisis actualizado del futuro de la gestión de los desechos sólidos. Banco Mundial, News. Recuperado el 23 de agosto de 2020. https://www.bancomundial.org/ es/news/immersive-story/2018/09/20/what-a-waste-an-updatedlook-into-the-future-of-solid-waste-management

Congreso de la República de Perú (2016). Decreto Legislativo $N^{\circ} 1278$.- Ley de Gestión Integral de Residuos Sólidos. Lima. Congreso de la República de Perú. https://sinia.minam.gob.pe/normas/ley-gestion-integralresiduos-solidos

Congreso de la República de Perú (2017). Decreto Supremo N014-2017-MINAM. - Reglamento del Decreto Legislativo $N^{\circ} 1278$, Decreto Legislativo que aprueba la Ley de Gestión Integral de Residuos Sólidos. Lima. Congreso de la República de Perú. https://sinia.minam.gob.pe/normas/ reglamento-decreto-legislativo-ndeg-1278-decreto-legislativo-que- 
DiRECTRICES QUE INFLUYEN EN EL «PROGRAMA DE SEGREgación EN LA FUENTE Y RECOLECCióN Selectiva de Residuos Sólidos» De las Municipalidades de SAN Martín De Porres y Ate

aprueba\#: :text=El\%20 presente $\% 20$ dispositivo $\% 20$ normativo $\% 20$ tiene,comprende\%20la\%20minimizaci\%C3\%B3n\%20de\%20la

Defensoría del Pueblo. (2019). Informe Defensorial $\mathrm{N}^{\circ} 181_{\text {¿Dónde va nuestra }}$ basura? https://www.defensoria.gob.pe/informes/informe-defensorialno-181-donde-va-nuestra-basura/

Espejo, K. (04 de noviembre de 2016). Cuatro Iniciativas municipales en favor del medio ambiente. Publimetro. https://www.publimetro.pe/ actualidad/2016/11/04/cuatro-iniciativas-municipales-favor-medioambiente-52396-noticia/

Fernández, A. (2005). La Gestión Integral de los Residuos Sólidos Urbanos en el Desarrollo Sostenible Local. Revista Cubana de Química, XVII (3), 35-39. https://www.redalyc.org/pdf/4435/443543687013.pdf

Grupo de Trabajo Multisectorial (2008). Diagnóstico Ambiental del Perú. Lima: MINAM. https://sinia.minam.gob.pe/documentos/diagnosticoambiental-peru

Huiman, A. (2018). Evaluación de la actividad de reciclaje en Lima Norte. Revista del Instituto de Investigación de la Facultad De Ingeniería Geológica, Minera, Metalúrgica y Geográfica, 21(42): 47-54. https://doi.org/10.15381/iigeo. v21i42.15786

Huiman, A. (26 de agosto de 2017). Hacia dónde va el reciclaje en el Perú. INTEPUCP, editorial. https://inte.pucp.edu.pe/editoriales/hacia-donde-vareciclaje-peru/

Ibáñez, J. y Corroppoli, M. (2002). Valorización de Residuos Sólidos Urbanos. (Anuario). Universidad Nacional de la Patagonia San Juan Bosco. Comodoro. http://www.ingenieroambiental.com/4014/valorizaresiduos.pdf

INEI (2007). Censos Nacionales 2007: XI de Población y VI de Vivienda - Cuadros Estadísticos. http://censos.inei.gob.pe/cpv2007/tabulados/

INEI (2015). Perú: Anuario de Estadísticas Ambientales 2015. Lima: INEI. https:// www.inei.gob.pe/media/MenuRecursivo/publicaciones_digitales/Est/ Lib1342/

INEI (2019). Perú: Anuario de Estadísticas Ambientales 2019. Lima: INEI. https:// www.inei.gob.pe/media/MenuRecursivo/publicaciones_digitales/Est/ Lib1704/libro.pdf

Latorre, A. (2016). La gestión compartida: elemento fundamental en la implementación de «Programas de segregación en la fuente y la recolección selectiva de los residuos sólidos» en las municipalidades de Lima Metropolitana. Presentado como parte del curso Investigación Académica, EEGGLL, PUCP. https://www.disa.com.pe/wp-content/ uploads/2017/12/Monograf\%C3\%ADa-Final-Alejandro-LatorreAlburquerque.pdf 
Lima Cómo Vamos (2019). Lima y Callao según sus ciudadanos: Décimo Informe Urbano de Percepción sobre Calidad de Vida en la Ciudad. http://www. limacomovamos.org/wp-content/uploads/2019/11/Encuesta-2019_ web.pdf

MINAM (1 de mayo de 2018). En el Perú solo se recicla el 1.9\% del total de residuos sólidos reaprovechables. Sinia, Novedades. Recuperado el 23 de agosto de 2020. https://sinia.minam.gob.pe/novedades/peru-solo-se-recicla-19total-residuos-solidos-reaprovechables

MINAM (2015). Guía metodológica para elaborar e implementar un Programa de Segregación en la Fuente y Recolección Selectiva de Residuos Sólidos Municipales. http://sial.segat.gob.pe/documentos/guia-metodologicaelaborar-implementar-un-programa-segregacion-fuente

Ministerio de Economía y Finanzas (s.f.). Programa de Incentivos a la Mejora de la Gestión Municipal - PI. https://www.mef.gob.pe/es/incentivos-paragobiernos-locales-y-regionales/programas-de-incentivos-municipalesa-la-mejora-de-la-gestion-municipal

Mulato, K. (2019). Desarrollo sostenible y el programa de segregación en la fuente y recolección selectiva de residuos sólidos del distrito de San Martín de Porres, Lima (tesis de pregrado para optar el título profesional de Ingeniera Ambiental). Universidad Continental, Huancayo. https://hdl. handle.net/20.500.12394/7035

Municipalidad de Ate (2016). Programa de Segregación y Recolección Selectiva de Residuos Sólidos en la Fuente del Distrito de Ate 2016. http://www. muniate.gob.pe/ate/planeamientoOrganizacion.php\#residuo\&id_ tema $=103 \&$ Ver $=$

Municipalidad de Ate (2017). Informe de Implementación de la Segregación en la Fuente y Recolección Selectiva de Residuos Sólidos. http://www.muniate. gob.pe/ate/files/transparencia/GERENCIA\%20DE\%20GESTION\%20 AMBIENTAL/SUB\%20GERENCIA\%20DE\%20GESTION\%20Y\%20 MANEJO\%20DE\%20RESIDUOS\%20SOLIDOS/

Municipalidad de San Martín de Porres (2016). Programa de Segregación en la Fuente y Recolección Selectiva de Residuos Sólidos 2015 en el distrito de San Martín de Porres. http://www.mdsmp.gob.pe/data_files/da-05-2015.pdf Municipalidad de San Martín de Porres (2018). Plan Anual de Segregación en la Fuente y Recolección Selectiva de Residuos Sólidos Municipales Inorgánicos 2018. http://www.mdsmp.gob.pe/data_files/rg_0534_2018.pdf

Municipalidad Metropolitana de Lima (2014). Plan Integral de Gestión Ambiental de Residuos Sólidos de Provincia de Lima. https://sinia.minam.gob.pe/ download/file/fid/64766

Recíclame Perú (19 de octubre de 2015). Incluyendo al Reciclaje en Ate [publicación de estado Facebook]. Recuperado de https://www.facebook.com/ ReciclamePlaneta/posts/715143318517918/ 
DiRECTRICES QUE INFLUYEN EN EL «PROGRAMA DE SEGREgación EN LA FUENTE Y RECOLECCióN Selectiva de Residuos Sólidos» de las Municipalidades de San Martín de Porres y Ate

Rentería, J. y Zeballos, M. (2014). Propuesta de Mejora para la gestión estratégica del Programa de Segregación en la Fuente y Recolección Selectiva de Residuos Sólidos Domiciliarios en el distrito de Los Olivos (tesis de pregrado para optar el título profesional de e Licenciado en Gestión, con mención Gestión Empresarial presentada). Lima, PUCP. http://tesis.pucp.edu.pe/ repositorio/bitstream/handle/20.500.12404/6285/RENTERIA_JOSE_ ZEBALLOS_MARIA_PROPUESTA_MEJORA.pdf?sequence=1\&isAllowed=y

Ruiz, A. (2010). Por la Ruta del Reciclaje en el Perú, Estudio Socioeconómico de la cadena del reciclaje. SEGAT, Sial Trujillo. http://sial.segat.gob.pe/ documentos/presentacion-ruta-reciclaje-peru-estudio-socioeconomicocadena

Sandoval, P. (13 de febrero de 2016). Solo 4\% de 8.468 toneladas diarias de basura se recicla en Lima. El Comercio. Recuperado el 23 de agosto de 2020. https://elcomercio.pe/lima/4-8-468-toneladas-diarias-basura-reciclalima-272561-noticia/?ref=ecr 